\title{
Non-Markovian Dynamics of Quantum Open Systems Embedded in a Hybrid Environment
}

\author{
Xinyu Zhao ${ }^{1}$, Wufu $\mathrm{Shi}^{1}$, J. Q. You ${ }^{2,3}$, Ting $\mathrm{Yu}^{1,2,4 *}$ \\ ${ }^{1}$ Department of Physics and Engineering Physics, Stevens Institute of Technology, Hoboken, \\ New Jersey 07030, USA \\ ${ }^{2}$ Beijing Computational Science Research Center, Beijing 100094, China \\ ${ }^{3}$ Synergetic Innovation Center of Quantum Information and Quantum Physics, University \\ of Science and Technology of China, Hefei, Anhui 230026, China \\ ${ }^{4}$ School of Physics and Optoelectronic Engineering, Yangtze University, Jingzhou 434023,
} China

\begin{abstract}
Quantum systems of interest are typically coupled to several quantum channels (more generally environments). In this paper, we develop an exact stochastic Schrödinger equation for an open quantum system coupled to a hybrid environment containing both bosonic and fermionic particles. Such a stochastic differential equation may be obtained directly from a microscopic model through employing a classical complex Gaussian noise and a non-commutative fermionic noise to simulate the hybrid bath. As an immediate application of our developed stochastic approach, we show that the evolution of the reduced density matrix can be derived by taking the average over both the bosonic noise and the fermionic noise. Three specific examples are given in this paper to illustrate that the hybrid quantum trajectory is fully consistent with the standard quantum mechanics. Our examples also shed new light on the special features exhibited by the fermionic bath and bosnoic bath.
\end{abstract}

Keywords: Open Quantum System, Non-Markovian, Stochastic

\section{Introduction}

A quantum system, when it is not isolated, can be in contact with several types of environments. Physically, such open quantum systems like an electron relaxation in a solid may interact with a bosonic system and be coupled to some fermionic systems at the same time [1, 2, 3]. In a similar manner, one can recognize that an atomic system of interest can be coupled to both classical laser fields and quantized radiation fields [4]. Therefore, a hybrid quantum open system

${ }^{*}$ Corresponding author's email: Ting.Yu@stevens.edu 


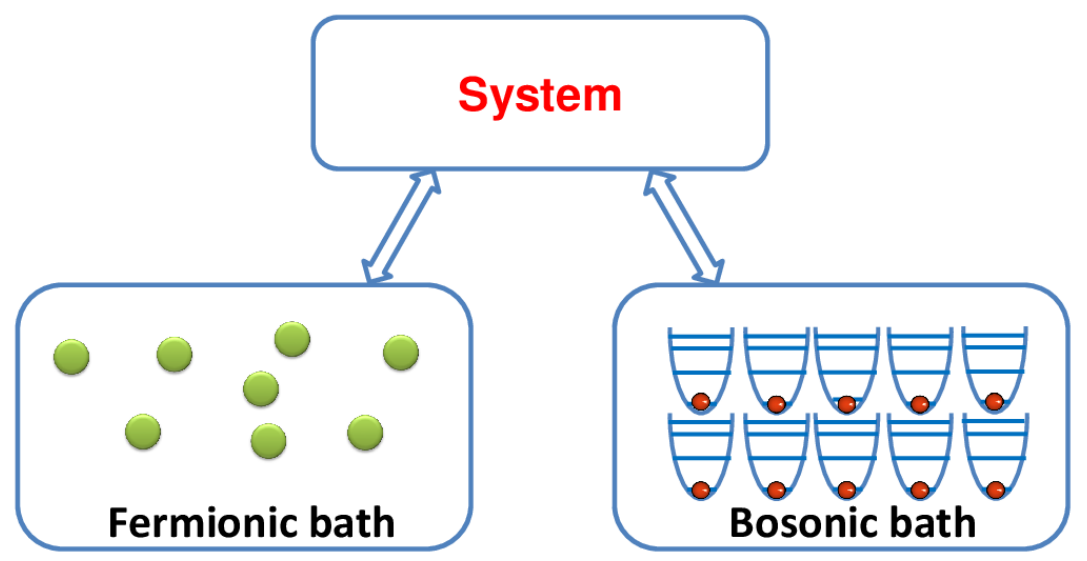

Figure 1: (Color online) Schematic diagram of a quantum system coupled to a fermionic bath and a bosonic bath simultaneously.

theory is potentially useful since it provides a systematic approach to dealing with the dynamics of an open system coupled to multiple environments in a direct manner. Fundamentally, the dynamics of open quantum systems embedded in one or more environments has attracted the wide-spread interest in recent years [5, $6,6,7,[8]$. On the one hand, the temporal behaviours of quantum open systems are essential for understanding many fundamental issues of quantum theory such as quantum dissipation and decoherence [9, 10, 11, 12, 13, 14, 15]. On the other hand, many novel applications based on quantum devices also require a better understanding on the interaction between the quantum system of interest and its environment in order to manipulate and control the system's dynamics [16, 17]. Although a realistic environment can be very complicated, it is typically composed of bosons and fermions. For a bosonic bath, a set of powerful tools have been developed to investigate the open system dynamics, such as path integral approach [18, 19], master equation approach 20, 21, 22, 23], and Markov and non-Markovian quantum trajectory approach [25, 24, 26, 27, 28]. For fermionic bath, similar tools have also been developed, including scattering theory [29], non-equilibrium Green's function approach 30], and fermionic path integral [31, 32]. Notably, the fermionic quantum state diffusion equations have been developed recently [33, 34, 35]. Although bosonic and fermionic formalisms share many similarities, a unified description of both types of baths is still useful for the purpose of direct applications.

In this paper, we will consider a hybrid case that the environment is composed of both bosons and fermions as shown in Fig. 1. Of many applications is the primary example of quantum dot model where the quantum dots may interact with two fermionic reservoirs (source and drain) and other agents such as a phonon bath. In this context, the dynamics of the quantum dot system is deter- 
mined by both the fermionic reservoirs and the bosonic bath. The theoretical approach to be developed in this paper will be capable of taking into account of the environmental effects arising from both types of environments. We shall show that the non-Markovian quantum state diffusion (NMQSD) approach is applicable to this extended case. In this method, the dynamic evolution of the open system is decomposed into an ensemble of quantum trajectories of pure states, and the reduced density matrix is described by the statistical average over these generated trajectories. It is worth noting the fact that this method is well developed for both bosonic bath and fermionic bath, and the method becomes one of the most hopeful candidates to solve the hybrid bath problem. For the case of bosonic bath, several interesting physical systems have been studied in the past fifteen years [26, 27, 28, 36, 37]. As a fundamentally theoretical study, it has been developed from solving single-particle system to solving many-body systems [8, 14, 15]. Furthermore, as a computing tool in real applications [38, 39], the NMQSD approach also showed its potential value in many interesting problems including precision quantum measurement [40], quantum control dynamics [41], and quantum biology [42]. In either the bosonic or fermionic NMQSD approach, the central idea is to encode all the influences of the environments on the system into a set of classical random variables forming a stochastic process. Taking the statistic average over the stochastic variables is equivalent to taking the partial trace over the environment to obtain the reduced density matrix. The difference between bosonic and fermionic approaches is that a bosonic bath can be represented by a complex Gaussian noise, while the fermionic bath is simulated by a non-commutative Grassmann noise. For a hybrid bath, an exact dynamical equation will typically contain both the complex Gaussian noise and Grassmann Gaussian noise.

The paper is organized as follows. In Sec. 2 we describe a model of hybrid bath and point out some new features arising from the hybrid bath case where the fermionic bath is assumed to commutes or anti-commutes with the system of interest. In Sec. 3] we analyze the commutative case with two examples. First, a general NMQSD equation and the corresponding master equation are derived. Then, we examine our general formalism by exactly solving a simple example of the single qubit dissipative model. It is shown that, as expected, the result predicted by the newly developed NMQSD approach is identical to the solution based on the ordinary quantum mechanics. Moreover, the two-qubit dissipative model is also studied for the hybrid bath case. Sec. 4 is devoted to investigating the anti-commutative case. Again, a general NMQSD approach can be developed. As an important example, we show how to use the new approach to study the Anderson model in the hybrid bath context. We identify the different impacts of fermionic bath and bosonic bath on the system dynamics. We conclude in Sec. 5 . 


\section{Two Types of Hybrid Bath: Commutative and Anti-commutative}

An open system embedded in a hybrid bath may be described by the following Hamiltonian

$$
H_{t o t}=H_{S}+H_{F B}+H_{B B}+H_{F I}+H_{B I},
$$

where $H_{S}$ describes the Hamiltonian of the system,

$$
H_{B B}=\sum_{r} \Omega_{r} b_{r}^{\dagger} b_{r}, H_{F B}=\sum_{k} \epsilon_{k} c_{k}^{\dagger} c_{k},
$$

are the bosonic bath and the fermionic bath respectively, where " $b_{r} "$ and " $c_{k}$ " are the annihilation operators for a single mode of bosonic bath and fermionic bath respectively. The interaction between system and two baths is given by

$$
H_{B I}=\sum_{r} \lambda_{r} b_{r}^{\dagger} L_{b}+\text { H.c., } H_{F I}=\sum_{k} \mu_{k} c_{k}^{\dagger} L_{f}+\text { H.c., }
$$

where $L_{b}$ and $L_{f}$ are the bosonic and fermionic coupling operators. Typically, the bosonic bath commutes with both the fermionic bath and the system no mater the system is composed of fermions or bosons. However, the commutation relation between the system and the fermionic bath could fall into two different categories. Depending on the commutation relation between the system and the fermionic bath (commutative or anti-commutative), the physical model for this Hamiltonian and the technique of solving this model are totally different. Therefore, we need to develop two parallel schemes to deal with these two different cases.

Case 1: System commutes with fermionic bath.

The Hamiltonian (11) for this case typically describes an effective fermionic bath. For example, a spin-chain bath can be transformed into an effective fermionic bath by using the Jordan-Wigner transformation and the Fourier transform [33, 43]. After the transformation, $t$ hese effective fermions satisfying fermionic commutation relations. However, since the original spins living in a Hilbert space separated from the system degree of freedom, they all commute with the system. Therefore, in the case of effective fermionic bath, the creation and annihilation operators $c_{k}^{\dagger}$ and $c_{k}$ will commute with any operators living in the Hilbert space of the system $H_{S}$.

Case 2: System anti-commutes with fermionic bath.

The anti-commutative case naturally arises when both the system and the bath are composed of a set of electrons. A well-known example is a quantum dot connected to a source and a drain reservoirs, where the system Hamiltonian of this model is $H_{S}=\omega_{d} d^{\dagger} d$. Obviously, the annihilation operator " $d$ " for the system and the operators " $c_{k}$ " for the bath satisfy the anti-commutation relation $\left\{d, c_{k}\right\}=0$ and $\left\{d^{\dagger}, c_{k}=0\right\}$.

We will develop two different schemes in the following sections for the two cases described above. Several specific examples are provided. 


\section{Commutative Case}

\subsection{General Stochastic Schrödinger Equation}

First, we consider the commutative case. In this case, the total Hamiltonian can be transformed into the interaction picture as

$$
H_{\text {tot }}^{i n t}=H_{S}+\left(\sum_{k} \mu_{k} c_{k}^{\dagger} L_{f} e^{i \epsilon_{k} t}+\sum_{r} \lambda_{r} b_{r}^{\dagger} L_{b} e^{i \Omega_{r} t}+\text { H.c. }\right) .
$$

By introducing multi-mode bosonic coherent states and fermionic coherent states

$$
\begin{aligned}
& |z\rangle=\prod_{r} \exp \left\{z_{r} b_{r}^{\dagger}\right\}|0\rangle, \\
& |\xi\rangle=\prod_{k}\left(1-\xi_{k} c_{k}^{\dagger}\right)|0\rangle,
\end{aligned}
$$

the stochastic state vector can be defined as

$$
\left|\psi_{t}\left(z^{*}, \xi^{*}\right)\right\rangle=\left\langle z^{*}, \xi^{*} \mid \psi_{\text {tot }}(t)\right\rangle .
$$

Throughout the paper, we will use the short-notation $\left|\psi_{t}\right\rangle \equiv\left|\psi_{t}\left(z^{*}, \xi^{*}\right)\right\rangle$ if no confusion arises. Because of the different properties of bosonic coherent states and fermionic coherent states [44], the noise variables introduced here are rather different. For bosonic coherent states, $z_{r}$ is an ordinary complex variable, while for fermionic coherent states, $\xi_{k}$ is a Grassmann variable satisfying anticommutative relations $\left\{\xi_{i}, \xi_{j}\right\}=0$. Starting with the Schrödinger equation for the total system, one can derive the dynamic equation for the stochastic state vector,

$$
\begin{aligned}
\frac{\partial}{\partial t}\left|\psi_{t}\right\rangle= & -i\left\langle z^{*}, \xi^{*}\left|H_{t o t}^{i n t}(t)\right| \psi_{t o t}(t)\right\rangle \\
= & {\left[-i H_{S}+L_{f} \xi_{t}^{*}-L_{f}^{\dagger} \int d s K_{f}(t, s) \frac{\delta_{l}}{\delta \xi_{s}^{*}}\right.} \\
& \left.+L_{b} z_{t}^{*}-L_{b}^{\dagger} \int d s K_{b}(t, s) \frac{\delta}{\delta z_{s}^{*}}\right]\left|\psi_{t}\right\rangle,
\end{aligned}
$$

where $K_{b}(t, s)=\sum_{r} \lambda_{r}^{2} e^{-i \Omega_{r}(t-s)}$ and $K_{f}(t, s)=\sum_{k} \mu_{k}^{2} e^{-i \epsilon_{k}(t-s)}$ are the correlation functions for the bosonic bath and the fermionic bath, respectively. The equation (8) is the fundamental equation governing the dynamics of the stochastic state vector $\left|\psi_{t}\right\rangle$. Note that this equation contains two types of noises as

$$
\begin{aligned}
& z_{t}^{*}=-i \sum_{r} z_{r}^{*} e^{i \Omega_{r} t}, \\
& \xi_{t}^{*}=-i \sum_{k} \xi_{k}^{*} e^{i \epsilon_{k} t},
\end{aligned}
$$


where $z_{t}^{*}$ is a complex Gaussian process and $\xi_{t}^{*}$ is a Grassmann stochastic process. They satisfy the following statistical relations

$$
\begin{aligned}
& \left\langle z_{t}\right\rangle_{b}=\left\langle z_{t}^{*}\right\rangle_{b}=0,\left\langle z_{t} z_{s}^{*}\right\rangle_{b}=K_{b}(t, s), \\
& \left\langle\xi_{t}\right\rangle_{f}=\left\langle\xi_{t}^{*}\right\rangle_{f}=0,\left\langle\xi_{t} \xi_{s}^{*}\right\rangle_{f}=K_{f}(t, s) .
\end{aligned}
$$

The statistical averages over both the complex noise and Grassmann noises are defined as $\langle\cdot\rangle_{b}=\int \prod_{r} \frac{1}{\pi} e^{-\left|z_{r}\right|^{2}} d z_{r}^{2}[\cdot]$ and $\langle\cdot\rangle_{f}=\int \prod_{k} d \xi_{k}^{*} d \xi_{k} e^{-\xi_{k}^{*} \xi_{k}}[\cdot]$, respectively.

In order to solve Eq. (8), one has to deal with the functional derivatives. Similar to the technique used in Refs. [27, 34, 33, 35], we can always replace these functional derivatives by some time-dependent operators $O$ and $Q$ as

$$
\begin{aligned}
\frac{\delta}{\delta z_{s}^{*}}\left|\psi_{t}\right\rangle & =O\left(t, s, z^{*}, \xi^{*}\right)\left|\psi_{t}\right\rangle, \\
\frac{\delta}{\delta \xi_{s}^{*}}\left|\psi_{t}\right\rangle & =Q\left(t, s, \xi^{*}, z^{*}\right)\left|\psi_{t}\right\rangle .
\end{aligned}
$$

Then, the NMQSD equation (8) can be written in a more compact form,

$$
\frac{\partial}{\partial t}\left|\psi_{t}\right\rangle=\left[-i H_{s}+L_{f} \xi_{t}^{*}-L_{f}^{\dagger} \bar{Q}+L_{b} z_{t}^{*}-L_{b}^{\dagger} \bar{O}\right]\left|\psi_{t}\right\rangle
$$

where $\bar{O}\left(t, z^{*}, \xi^{*}\right)=\int_{0}^{t} K_{b}(t, s) O\left(t, s, z^{*}, \xi^{*}\right) d s, \bar{Q}\left(t, z^{*}, \xi^{*}\right)=\int_{0}^{t} K_{f}(t, s) Q\left(t, s, z^{*}, \xi^{*}\right) d s$. If these time-dependent operators $O$ and $Q$ can be determined, the NMQSD equation will take a time-local form and the equation can be solved numerically in a more straightforward way. The consistency condition may be employed,

$$
\begin{aligned}
& \frac{\partial}{\partial t} \frac{\delta}{\delta z_{s}^{*}}\left|\psi_{t}\right\rangle=\frac{\delta}{\delta z_{s}^{*}} \frac{\partial}{\partial t}\left|\psi_{t}\right\rangle, \\
& \frac{\partial}{\partial t} \frac{\delta}{\delta \xi_{s}^{*}}\left|\psi_{t}\right\rangle=\frac{\delta}{\delta \xi_{s}^{*}} \frac{\partial}{\partial t}\left|\psi_{t}\right\rangle,
\end{aligned}
$$

and gives rise to

$$
\begin{aligned}
\frac{\partial}{\partial t} O= & {\left[-i H_{s}+L_{f} \xi_{t}^{*}-L_{f}^{\dagger} \bar{Q}+L_{b} z_{t}^{*}-L_{b}^{\dagger} \bar{O}, O\right] } \\
& -L_{b}^{\dagger} \frac{\delta}{\delta z_{s}^{*}} \bar{O}-L_{f}^{\dagger} \frac{\delta}{\delta z_{s}^{*}} \bar{Q} \\
\frac{\partial}{\partial t} Q= & {\left[-i H_{s}, Q\right]-\left\{L_{f} \xi_{t}^{*}, Q\right\}+\left[L_{b} z_{t}^{*}, Q\right] } \\
& -L_{f}^{\dagger} \bar{Q}\left(-\xi^{*}\right) Q+Q L_{f}^{\dagger} \bar{Q}-L_{b}^{\dagger} \bar{O}\left(-\xi^{*}\right) Q \\
& +Q L_{b}^{\dagger} \bar{O}-L_{b}^{\dagger} \frac{\delta_{l}}{\delta \xi_{s}^{*}} \bar{O}-L_{f}^{\dagger} \frac{\delta_{l}}{\delta \xi_{s}^{*}} \bar{Q}
\end{aligned}
$$


with the initial conditions

$$
\begin{aligned}
& O\left(t, t, z^{*}, \xi^{*}\right)=L_{b}, \\
& Q\left(t, t, z^{*} \xi^{*}\right)=L_{f} .
\end{aligned}
$$

Given these conditions, the $O$ and $Q$ operators can be fully determined, as a result, the NMQSD equation (15) becomes more useful for the analytical purpose and numerical simulations. However, A single solution of the NMQSD equation can not fully describe the dynamic evolution of the system. Actually, it only gives one possible realization of many possible solutions of the NMQSD equation corresponding a specific sample path taken by the stochastic process $z_{t}^{*}$ and $\xi_{t}^{*}$. In order to obtain the full picture of the evolution of the system, we need to reproduce the reduced density matrix from the stochastic state vector $\left|\psi_{t}\right\rangle$ as

$$
\rho(t)=\left\langle\left\langle P_{t}\right\rangle_{f}\right\rangle_{b},
$$

where $P_{t} \equiv\left|\psi_{t}\left(z^{*}, \xi^{*}\right)\right\rangle\left\langle\psi_{t}\left(z^{*},-\xi^{*}\right)\right|$ is the stochastic density operator. Given the relation Eq. (22), the physical meaning of the NMQSD equation becomes clear. By choosing a random realization of the noises $z_{t}^{*}$ and $\xi_{t}^{*}$ (reflecting the states of the environment), the evolution of the reduced density matrix is decomposed into many pure-state quantum trajectories $\left|\psi_{t}\right\rangle$. However, taking the statistical average over all of these trajectories, the reduced density matrix is reproduced. Therefore, the complicated properties of the environment are all encoded into noise functions $z_{t}^{*}$ and $\xi_{t}^{*}$, so that tracing out the environment is equivalent to taking average over all the realizations of the noises. Based on the relation (22), the master equation can be derived as

$$
\begin{aligned}
\frac{d}{d t} \rho= & -i\left[H_{S}, \rho\right]+\left[L_{b},\left\langle\left\langle P_{t} \bar{O}^{\dagger}\right\rangle_{f}\right\rangle_{b}\right]+\left[\left\langle\left\langle\bar{O} P_{t}\right\rangle_{f}\right\rangle_{b}, L_{b}^{\dagger}\right] \\
& +\left[L_{f},\left\langle\left\langle P_{t} \bar{Q}^{\dagger}(-\xi)\right\rangle_{f}\right\rangle_{b}\right]+\left[\left\langle\left\langle\bar{Q} P_{t}\right\rangle_{f}\right\rangle_{b}, L_{f}^{\dagger}\right],
\end{aligned}
$$

where the Novikov theorem for fermionic case [33] and bosonic case 27] are used in the derivation. Although taking the statistical averages $\langle\cdot\rangle_{b}$ and $\langle\cdot\rangle_{f}$ are not simple in the general case, there is still a special case. When the operators $O$ and $Q$ are noise-independent, the master equation can be written in a simpler form as

$$
\frac{d}{d t} \rho=-i\left[H_{S}, \rho\right]+\left\{\left[\bar{Q} \rho, L_{f}^{\dagger}\right]+\left[\bar{O} \rho, L_{b}^{\dagger}\right]+\text { H.c. }\right\},
$$

Actually, this special case is very common in many interesting models [26, 33, 27, 15] in which the exact $O$ or $Q$ operators just contain no noises. Moreover, in general case, we can still expand $O$ and $Q$ into functional series and only taking the first term (with zeroth order of noise variables) of the expansions as $O\left(t, s, z^{*}, \xi^{*}\right) \approx O^{(0)}(t, s)$ and $Q\left(t, s, z^{*}, \xi^{*}\right) \approx Q^{(0)}(t, s)$. This approximation is called the zeroth order approximation [27]. The validity and accuracy of this approximation is analyzed in Ref. [45]. Actually, the accuracy is proved to be much better than the weak coupling approximation. 


\subsection{Example 1: Two qubits in a hybrid bath}

In order to illustrate the NMQSD approach for a hybrid bath we discussed above, we will solve some particular examples in details. In the first example, we will consider a two-qubit system interacting with two dissipative baths, one is bosonic and the other fermionic. From this example, we show that dynamic equation for a hybrid bath is not the simple combination of a fermionic bath and a bosonic bath. The cross-terms in $O$ and $Q$ operators reflect the correlation between two baths through interaction with the system of interest. In the general model described by Eqs. (1, 3), the two qubits example is the special case that

$$
\begin{gathered}
H_{S}=\frac{\omega}{2}\left(\sigma_{z}^{A}+\sigma_{z}^{B}\right), \\
L_{b}=L_{f}=\sigma_{-}^{A}+\kappa_{B} \sigma_{-}^{B} .
\end{gathered}
$$

where $\kappa_{B}$ is a parameter describing the coupling strength between the second qubit and the baths. We will first investigate the case with $\kappa_{B}=1$ in this subsection. A special case with $\kappa_{B}=0$ will be considered later, which means the second qubit evolves independently from the other part of the total system and the model reduces to a single qubit case. Given this specific model, the NMQSD equation can be formally written as

$$
\begin{aligned}
\frac{\partial}{\partial t}\left|\psi_{t}\right\rangle= & {\left[-i \frac{\omega}{2}\left(\sigma_{z}^{A}+\sigma_{z}^{B}\right)+\left(\sigma_{-}^{A}+\sigma_{-}^{B}\right)\left(\xi_{t}^{*}+z_{t}^{*}\right)\right.} \\
& \left.-\left(\sigma_{+}^{A}+\sigma_{+}^{B}\right)(\bar{Q}+\bar{O})\right]\left|\psi_{t}\right\rangle .
\end{aligned}
$$

In fact, it is instructive to compare this dynamic equation for a hybrid bath with the model that two qubits interact with either a single bosonic bath or a single fermionic bath. For a single bath, the NMQSD equation should be $\frac{\partial}{\partial t}\left|\psi_{t}\right\rangle=$ $\left[-i H_{S}+L_{b} z_{t}^{*}-L_{b}^{\dagger} \bar{O}\right]\left|\psi_{t}\right\rangle$ (bosonic [7]) or $\frac{\partial}{\partial t}\left|\psi_{t}\right\rangle=\left[-i H_{S}+L_{f} \xi_{t}^{*}-L_{f}^{\dagger} \bar{Q}\right]\left|\psi_{t}\right\rangle$ (fermionic [33]). It seems that Eq. (27) is nothing more than a direct summation of a fermionic bath and a bosonic bath. However, more information is encoded in the $O$ and $Q$ operators. According to Eqs. (18, 19), the exact $O$ and $Q$ operators can be determined as

$$
\begin{aligned}
O= & f_{1}(t, s) O_{1}+f_{2}(t, s) O_{2}+i \int_{0}^{t} d s^{\prime} f_{3}\left(t, s, s^{\prime}\right) z_{s^{\prime}}^{*} O_{3} \\
& +i \int_{0}^{t} d s^{\prime} f_{4}\left(t, s, s^{\prime}\right) \xi_{s^{\prime}}^{*} O_{4}, \\
Q= & g_{1}(t, s) Q_{1}+g_{2}(t, s) Q_{2}+i \int_{0}^{t} d s^{\prime} g_{3}\left(t, s, s^{\prime}\right) z_{s^{\prime}}^{*} Q_{3} \\
& +i \int_{0}^{t} d s^{\prime} g_{4}\left(t, s, s^{\prime}\right) \xi_{s^{\prime}}^{*} Q_{4} .
\end{aligned}
$$


The basis operators are $O_{1}=\sigma_{-}^{A}+\sigma_{-}^{B}, O_{2}=\left(\sigma_{z}^{A}+\sigma_{z}^{B}\right)\left(\sigma_{-}^{A}+\sigma_{-}^{B}\right), O_{3}=O_{4}=$ $\sigma_{-}^{A} \sigma_{-}^{B}, Q_{1}=\sigma_{-}^{A}+\sigma_{-}^{B}, Q_{2}=\left(\sigma_{z}^{A}+\sigma_{z}^{B}\right)\left(\sigma_{-}^{A}+\sigma_{-}^{B}\right), Q_{3}=Q_{4}=\sigma_{-}^{A} \sigma_{-}^{B}$. The time-dependent coefficients satisfy the following relations

$$
\begin{aligned}
& \frac{\partial}{\partial t} f_{1}(t, s)=i \omega f_{1}+4 f_{1} F_{2}+4 f_{1} G_{2}+i F_{3}+i G_{3}, \\
& \frac{\partial}{\partial t} f_{2}(t, s)=i \omega f_{2}+f_{1}\left(4 F_{2}+4 G_{2}-F_{1}-G_{1}\right)-\frac{i}{2} F_{3} \\
& +f_{2}\left(2 F_{1}+2 G_{1}-4 F_{2}-4 G_{2}\right)-\frac{i}{2} G_{3}, \\
& \frac{\partial}{\partial t} f_{3}\left(t, s, s^{\prime}\right)=2 i \omega f_{3}+2 f_{1} F_{3}+2 f_{1} G_{3}-4 f_{2} F_{3} \\
& -4 f_{2} G_{3}+2 f_{3} F_{1}+2 f_{3} G_{1}, \\
& \frac{\partial}{\partial t} f_{4}\left(t, s, s^{\prime}\right)=2 i \omega f_{4}+2 f_{1} F_{4}+2 f_{1} G_{4}-4 f_{2} F_{4} \\
& -4 f_{2} G_{4}+2 f_{4} F_{1}+2 f_{4} G_{1}, \\
& \frac{\partial}{\partial t} g_{1}(t, s)=i \omega g_{1}+4 g_{1} F_{2}+4 g_{1} G_{2}+i F_{3}+i G_{3}, \\
& \frac{\partial}{\partial t} g_{2}(t, s)=i \omega g_{2}+g_{1}\left(4 F_{2}+4 G_{2}-F_{1}-G_{1}\right)-\frac{i}{2} F_{3} \\
& +g_{2}\left(2 F_{1}+2 G_{1}-4 F_{2}-4 G_{2}\right)-\frac{i}{2} G_{3}, \\
& \frac{\partial}{\partial t} g_{3}\left(t, s, s^{\prime}\right)=2 i \omega g_{3}+2 g_{1} F_{3}+2 g_{1} G_{3}-4 g_{2} F_{3} \\
& -4 g_{2} G_{3}+2 g_{3} F_{1}+2 g_{3} G_{1}, \\
& \frac{\partial}{\partial t} g_{4}\left(t, s, s^{\prime}\right)=2 i \omega g_{4}+2 g_{1} F_{4}+2 g_{1} G_{4}-4 g_{2} F_{4} \\
& -4 g_{2} G_{4}+2 g_{4} F_{1}+2 g_{4} G_{1},
\end{aligned}
$$

with the initial conditions

$$
\begin{gathered}
f_{1}(t, t)=g_{1}(t, t)=1, \quad f_{2}(t, t)=g_{2}(t, t)=0, \\
f_{3}\left(t, t, s^{\prime}\right)=f_{4}\left(t, t, s^{\prime}\right)=g_{3}\left(t, t, s^{\prime}\right)=g_{4}\left(t, t, s^{\prime}\right)=0, \\
g_{3}(t, s, t)=-4 i g_{2}(t, s), g_{4}(t, s, t)=-4 i g_{1}(t, s)+4 i g_{2}(t, s),
\end{gathered}
$$




$$
f_{3}(t, s, t)=f_{4}(t, s, t)=-4 i f_{2}(t, s) .
$$

where

$$
\begin{aligned}
F_{i}(t) & =\int_{0}^{t} K_{b}(t, s) f_{i}(t, s) d s, \quad(i=1,2) \\
G_{i}(t) & =\int_{0}^{t} K_{f}(t, s) g_{i}(t, s) d s, \quad(i=1,2) \\
F_{i}\left(t, s^{\prime}\right) & =\int_{0}^{t} K_{b}(t, s) f_{i}\left(t, s, s^{\prime}\right) d s, \quad(i=3,4) \\
G_{i}\left(t, s^{\prime}\right) & =\int_{0}^{t} K_{f}(t, s) g_{i}\left(t, s, s^{\prime}\right) d s, \quad(i=3,4)
\end{aligned}
$$

In this example, both $O$ and $Q$ operators contain the fermionic noise $\xi^{*}$, but the fermionic $Q$ operator also contains the bosonic noise $z^{*}$. Although the NMQSD equation (27) seems to be a direct summation of two individual baths, the $O$ and $Q$ operators in a hybrid bath are not a simple combination of these operators obtained in the single bath case (the exact $O$ or $Q$ operators for a single bosonic bath or a fermionic bath can be found in Ref. 7] and Ref. [33] respectively). Instead, there are many cross terms and they are coupled to each other reflecting the fact that the effect of a hybrid bath cannot be simply treated as the direct summation of a fermionic bath plus a bosonic bath. Through the system, two baths are also coupled indirectly. Such kind of indirect coupling can be also considered as interference between two independent baths which has been recently discussed in Ref. [46]. With the exact solution, it is possible to investigate the interference between fermionic bath and bosonic bath in the future research. Here, we just show one numerical result as an example to demonstrate that the interference between two baths can be dominant under certain conditions. In Fig. 2, we compare the time evolutions of the coefficients in $O$ and $Q$ operators. The functions $F_{i}^{\prime}(t)(i=3,4)$ are defined as $F_{i}^{\prime}(t)=$ $\int d s^{\prime} K_{b}\left(t, s^{\prime}\right) F_{i}\left(t, s^{\prime}\right)(i=3,4)$. Similarly, the functions $G_{i}^{\prime}(t)(i=3,4)$ are defined as $G_{i}^{\prime}(t)=\int d s^{\prime} K_{f}\left(t, s^{\prime}\right) G_{i}\left(t, s^{\prime}\right)(i=3,4)$. In the single bath case 7], $O$ operator should not contain fermionic noise term $i \int_{0}^{t} d s^{\prime} f_{4}\left(t, s, s^{\prime}\right) \xi_{s^{\prime}}^{*} O_{4}$. However, according to the numerical results for hybrid bath, the coefficient for fermionic noise term, $F_{4}^{\prime}(t)$ can be dominant under certain conditions. Similarly, in the $Q$ operator, the bosonic noise term $G_{3}^{\prime}(t)$ is also larger than $G_{1}(t)$ and $G_{2}(t)$. These results imply that the interference between two baths can be rather complicated and important. It is also worth noting that the results in Fig. 2 is obtained in a strongly non-Markovian environment. In a Markov case, $F_{1}(t)$ and $G_{1}(t)$ will be dominant. Thus, our exact treatment of the non-Markovian hybrid bath problem could be a valuable tool to study those properties in the future. 

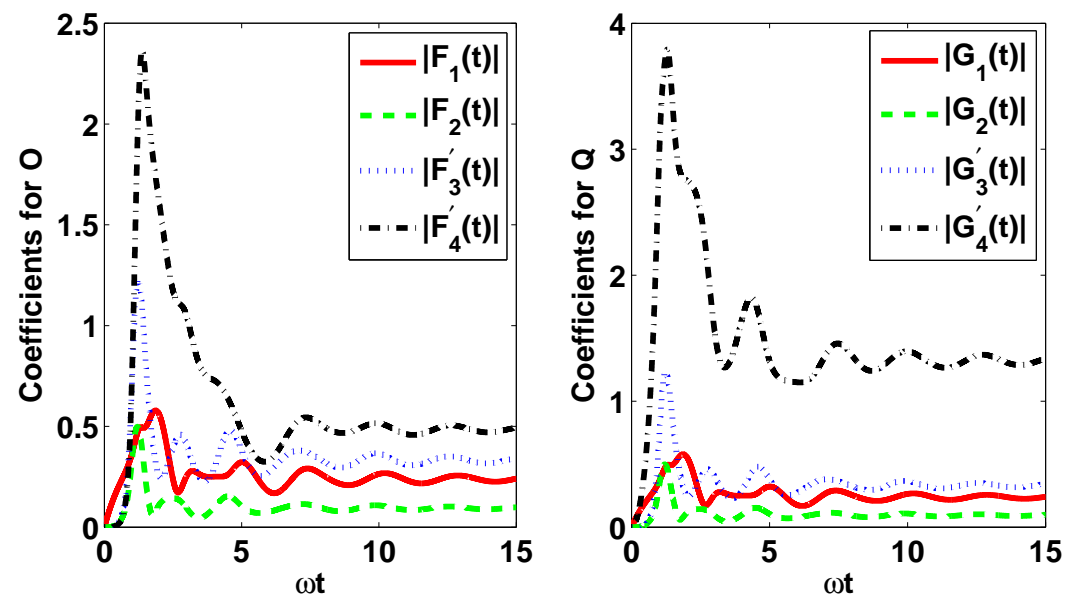

Figure 2: (Color online) Time evolution for the coefficients in $O$ and $Q$ operators. In the left subplot, the red (solid), green (dashed), blue (dash-dotted) and black (dash-dotted) curves are the absolute values of the coefficients $\left|F_{1}(t)\right|,\left|F_{2}(t)\right|,\left|F_{3}^{\prime}(t)\right|$, and $\left|F_{4}^{\prime}(t)\right|$, respectively. In the right subplot, those curves represent $\left|G_{1}(t)\right|,\left|G_{2}(t)\right|,\left|G_{3}^{\prime}(t)\right|$, and $\left|G_{4}^{\prime}(t)\right|$, respectively.

\subsection{Single qubit case: Consistency with ordinary quantum mechanics}

Although the $O$ and $Q$ operators in the two-qubit example are rather complicated, it is still possible to find a simple form when a special case- the single qubit case is considered, namely $\kappa_{B}=0$. In this case, the second qubit evolves independently from all the other parts, so that it can be removed in the interaction picture. Therefore, the NMQSD equation is reduced to

$$
\frac{\partial}{\partial t}\left|\psi_{t}\right\rangle=\left[-i \frac{\omega}{2} \sigma_{z}^{A}+\sigma_{-}^{A}\left(\xi_{t}^{*}+z_{t}^{*}\right)-\sigma_{+}^{A}(\bar{Q}+\bar{O})\right]\left|\psi_{t}\right\rangle,
$$

where the exact $O$ and $Q$ operators can be determined as

$$
O=Q=f(t, s) \sigma_{-}^{A} .
$$

The time-dependent coefficient $f(t, s)$ satisfies the equation

$$
\frac{\partial}{\partial t} f(t, s)=[i \omega+F(t)] f(t, s)
$$

where $F(t)=\int_{0}^{t}\left[K_{b}(t, s)+K_{f}(t, s)\right] f(t, s) d s$. Finally, the exact master equation for this model is derived as

$$
\frac{d}{d t} \rho=-i\left[H_{S}, \rho\right]+\left\{F(t)\left[\sigma_{-} \rho, \sigma_{+}\right]+\text {H.c. }\right\} .
$$

In general, the correlation functions $K_{b}(t, s)$ and $K_{f}(t, s)$ can be very complicated. However, here, we will use a special case to show that the result derived 
from NMQSD approach is consistent with the ordinary quantum mechanics. Consider the special case that there are only one boson and one fermion in the bosonic bath and fermionic bath respectively, i.e., $H_{F B}=\epsilon c^{\dagger} c, H_{B B}=\Omega b^{\dagger} b$. Therefore, the correlation functions is reduced to

$$
\begin{aligned}
& K_{b}(t, s)=\lambda^{2} e^{-i \Omega(t-s)}, \\
& K_{f}(t, s)=\mu^{2} e^{-i \epsilon(t-s)} .
\end{aligned}
$$

In the resonance case, $\omega=\epsilon=\Omega, \lambda=\mu$, the equation for $F(t)$ is

$$
\frac{\partial}{\partial t} F(t)=2 \lambda^{2}+F^{2}(t)
$$

The solution is

$$
F(t)=\sqrt{2} \lambda \tan (\sqrt{2} \lambda t) .
$$

From the master equation (49), the evolution of the off-diagonal element $\rho_{21}$ is

$$
\frac{d}{d t} \rho_{21}(t)=i \omega \rho_{21}-F^{*}(t) \rho_{21}
$$

Finally, the solution of $\rho_{21}(t)$ is

$$
\rho_{21}(t)=\rho_{21}(0) e^{i \omega t} \cos (\sqrt{2} \lambda t) .
$$

On the other hand, it is also straightforward to solve the whole system (system plus two "baths") with the standard Schrödinger equation since the total system only contains three particles. It is easy to confirm that solving the whole system gives the identical result as we obtained in Eq. (55) by using the NMQSD approach. It confirms that our NMQSD approach is indeed consistent with ordinary quantum mechanics as expected.

\subsection{Example 2: Single qubit with dephasing bosonic bath and dissipative fermionic bath}

In the second example, we will investigate the case that the system is coupled to the bosonic bath and fermionic bath in two different ways. The model we considered is given by

$$
\begin{gathered}
H_{S}=\frac{\omega}{2} \sigma_{z}, \\
L_{b}=\sigma_{z}, \quad L_{f}=\sigma_{-} .
\end{gathered}
$$

According to the general discussion in subsection 3.1, the NMQSD equation for this model can be written as

$$
\frac{\partial}{\partial t}\left|\psi_{t}\right\rangle=\left[-i \frac{\omega}{2} \sigma_{z}+\sigma_{-} \xi_{t}^{*}-\sigma_{+} \bar{Q}+\sigma_{z} z_{t}^{*}-\sigma_{z} \bar{O}\right]\left|\psi_{t}\right\rangle
$$


In this example, the $O$ and $Q$ operators contains infinite order of noise, therefore, for simplicity, we use the zeroth order functional expansion to give the approximate zeroth order operators $O^{(0)}$ and $Q^{(0)}$. By assuming all the terms associated with noises are zero [45], the zeroth order $O^{(0)}$ and $Q^{(0)}$ operators can be obtained from Eqs. (18, 19) as

$$
\begin{gathered}
O^{(0)}=\sigma_{z}, \\
Q^{(0)}=g(t, s) \sigma_{-},
\end{gathered}
$$

where the function $g(t, s)$ satisfies

$$
\frac{\partial}{\partial t} g(t, s)=[i \omega+G(t)] g(t, s),
$$

where $G(t)=\int_{0}^{t} g(t, s) K_{f}(t, s) d s$. Finally, the corresponding approximate master equation is

$$
\frac{d}{d t} \rho=-i\left[\frac{\omega}{2} \sigma_{z}, \rho\right]+\left\{G(t)\left[\sigma_{-} \rho, \sigma_{+}\right]+F(t)\left[\sigma_{z} \rho, \sigma_{z}\right]+\text { H.c. }\right\},
$$

where $F(t)=\int_{0}^{t} K_{b}(t, s) d s$. Different from the first example where the model can be solved exactly, we show how to use the zeroth order (for higher order expansion, see Ref. 45]) approximation to derive an approximate master equation in this second example. It is worth noting that Eq. (62) still contains incomplete non-Markovian information, although it is derived from the zeroth order approximation. In the Markov limit, the correlation functions $K_{b}(t, s)$ and $K_{f}(t, s)$ are all $\delta$-functions, and the coefficients are no longer time-dependent but reduced to constants. The zeroth-order approximation can partially capture the nonMarkovian features as a way to improve the Markov approximation. Actually, in the real application of the NMQSD approach, this systematic approximation method is shown to be very useful since the exact $O$ and $Q$ are often difficult to find in many realistic models. With this approximation approach, one can still solve these non-Markovian problems with satisfactory accuracy.

\section{Anti-commutative Case}

\subsection{General Stochastic Schrödinger Equation}

After discussing the commutative hybrid bath, we will consider the case that the system is assumed to anti-commutes with the fermionic bath, which often describes an electronic system such as a quantum dot system. Following a similar procedure, we can also derive the NMQSD equation for the anticommutative hybrid bath as

$$
\begin{aligned}
\frac{\partial}{\partial t}\left|\psi_{t}\right\rangle= & -i\left\langle z^{*}, \xi^{*}\left|H_{t o t}^{i n t}(t)\right| \psi_{t o t}(t)\right\rangle \\
= & {\left[-i H_{S}-L_{f} \xi_{t}^{*}-L_{f}^{\dagger} \int d s K_{f}(t, s) \frac{\delta_{l}}{\delta \xi_{s}^{*}}\right.} \\
& \left.+L_{b} z_{t}^{*}-L_{b}^{\dagger} \int d s K_{b}(t, s) \frac{\delta}{\delta z_{s}^{*}}\right]\left|\psi_{t}\right\rangle .
\end{aligned}
$$


Since the operators $L_{f}$ typically anti-commutes with the fermionic bath, the derivation is slightly different from the commutative case [35, 34, 33]. As a result, there is a minor difference between Eq. (81) and Eq. (63). The bosonic noise $z_{t}^{*}$, the fermionic niose $\xi_{t}^{*}$ and the corresponding correlation functions $K_{b}(t, s)$ and $K_{f}(t, s)$ are all defined in the same way. Similarly, we can also define the bosonic $O$ operator and the fermionic $Q$ operator, however, they satisfy different differential equations in the anti-commutative case as

$$
\begin{aligned}
\frac{\partial}{\partial t} O= & {\left[-i H_{s}-L_{f} \xi_{t}^{*}-L_{f}^{\dagger} \bar{Q}+L_{b} z_{t}^{*}-L_{b}^{\dagger} \bar{O}, O\right] } \\
& -L_{b}^{\dagger} \frac{\delta}{\delta z_{s}^{*}} \bar{O}-L_{f}^{\dagger} \frac{\delta}{\delta z_{s}^{*}} \bar{Q} \\
\frac{\partial}{\partial t} Q= & {\left[-i H_{s}, Q\right]+\left[L_{f} \xi_{t}^{*}, Q\right]+\left[L_{b} z_{t}^{*}, Q\right] } \\
& -L_{f}^{\dagger} \bar{Q} Q+Q L_{f}^{\dagger} \bar{Q}-L_{b}^{\dagger} \bar{O} Q+Q L_{f}^{\dagger} \bar{O} \\
& -L_{b}^{\dagger} \frac{\delta_{l}}{\delta \xi_{s}^{*}} \bar{O}+L_{f}^{\dagger} \frac{\delta_{l}}{\delta \xi_{s}^{*}} \bar{Q}
\end{aligned}
$$

with the initial conditions

$$
\begin{aligned}
& O\left(t, t, z^{*}, \xi^{*}\right)=L_{b}, \\
& Q\left(t, t, z^{*} \xi^{*}\right)=L_{f} .
\end{aligned}
$$

Then, the density matrix can be also reproduced as

$$
\rho(t)=\left\langle\left\langle P_{t}\right\rangle_{f}\right\rangle_{b}
$$

and the master equation can be derived as

$$
\begin{aligned}
\frac{d}{d t} \rho= & -i\left[H_{S}, \rho\right]+\left[L_{b},\left\langle\left\langle P_{t} \bar{O}^{\dagger}\right\rangle_{f}\right\rangle_{b}\right]+\left[\left\langle\left\langle\bar{Q} P_{t}\right\rangle_{f}\right\rangle_{b}, L_{b}^{\dagger}\right] \\
& +\left[L_{f},\left\langle\left\langle P_{t} \bar{Q}^{\dagger}(-\xi)\right\rangle_{f}\right\rangle_{b}\right]+\left[\left\langle\left\langle\bar{Q} P_{t}\right\rangle_{f}\right\rangle_{b}, L_{f}^{\dagger}\right] .
\end{aligned}
$$

In the derivation of the master equation, an anti-commutative version of the Novikov theorem [34, 35] has been used. It is different from either the commutative version of Novikov theorem for fermionic bath [33] or the one for bosonic bath [27]. Similarly, when $O$ and $Q$ are noise-independent, the master equation is reduced to

$$
\frac{d}{d t} \rho=-i\left[H_{S}, \rho\right]+\left\{\left[\bar{Q} \rho, L_{f}^{\dagger}\right]+\left[\bar{O} \rho, L_{b}^{\dagger}\right]+\text { H.c. }\right\} .
$$




\subsection{Example 3: Quantum dot in a hybrid bath}

In order to show the details of solving an anti-commutative hybrid bath problem, we consider a specific example that is the Anderson model in a bosonic

environment (see Ref. 47] for example). In this particular example the general Hamiltonian Eq. (11) becomes

$$
H_{S}=\varepsilon d^{\dagger} d,
$$

describing the quantum dot,

$$
H_{B}=\sum_{k, i=L, R}\left[\epsilon(k)-\mu_{i}\right] c_{k i}^{\dagger} c_{k i}+\sum_{r} \omega_{r} b_{r}^{\dagger} b_{r},
$$

describing the two fermionic baths (" $L$ " and " $R$ ") and one phonon bath, and

$$
H_{I}=\sum_{k, i=L, R} t_{k, i} c_{k i}^{\dagger} d+\text { H.c. }+\sum_{r} \lambda_{r}\left(d^{\dagger} d-\frac{1}{2}\right)\left(b_{r}+b_{r}^{\dagger}\right),
$$

describing the transport process between two fermionic bath and the dissipation process caused by a bosonic bath.

In the finite temperature case [36], we need to introduce two fictitious bath " $a_{L}$ " and " $a_{R}$ " with the negative eigen-frequencies as:

$$
\begin{aligned}
H= & H_{S}+\sum_{k, i=L, R}\left[\epsilon(k)-\mu_{i}\right] c_{k i}^{\dagger} c_{k i}+\left\{t_{k i} c_{k i}^{\dagger} d+\text { H.c. }\right\} \\
& +\sum_{r} \lambda_{r}\left(d^{\dagger} d-\frac{1}{2}\right)\left(b_{r}+b_{r}^{\dagger}\right)+\sum_{r} \Omega_{r} b_{r}^{\dagger} b_{r} \\
& +\sum_{k, i=L, R}-\left[\epsilon(k)-\mu_{i}\right] a_{k i}^{\dagger} a_{k i} .
\end{aligned}
$$

Then, performing the Bogoliubov transformation

$$
\begin{aligned}
& c_{k i}=\sqrt{1-\bar{n}_{k i}} c_{k i}^{\prime}+\sqrt{\bar{n}_{k i}} a_{k i}^{\prime \dagger} \quad(i=L, R), \\
& a_{k i}=\sqrt{1-\bar{n}_{k i}} a_{k i}^{\prime}-\sqrt{\bar{n}_{k i}} c_{k i}^{\prime \dagger} \quad(i=L, R),
\end{aligned}
$$

the Hamiltonian become

$$
\begin{aligned}
H= & H_{S}+\sum_{k, i=L, R}\left[\epsilon(k)-\mu_{i}\right] c_{k i}^{\prime \dagger} c_{k i}^{\prime} \\
& +\left\{t_{k i}\left(\sqrt{1-\bar{n}_{k i}} c_{k i}^{\prime \dagger}+\sqrt{\bar{n}_{k i}} a_{k i}^{\prime}\right) d+\text { H.c. }\right\} \\
& +\sum_{r} \lambda_{r}\left(d^{\dagger} d-\frac{1}{2}\right)\left(b_{r}+b_{r}^{\dagger}\right)+\sum_{r} \Omega_{r} b_{r}^{\dagger} b_{r} \\
& +\sum_{k, i=L, R}-\left[\epsilon(k)-\mu_{i}\right] a_{k i}^{\prime \dagger} a_{k i}^{\prime} .
\end{aligned}
$$


Redefining $\omega_{k i}=\epsilon(k)-\mu_{i}, g_{k i}=t_{k i} \sqrt{1-\bar{n}_{k i}}, f_{k i}=t_{k i} \sqrt{\bar{n}_{k i}}$, then, in the interaction picture, the Hamiltonian can be written as

$$
\begin{aligned}
H_{i n t}(t) & =H_{S}+\sum_{r} \lambda_{r}\left(d^{\dagger} d-\frac{1}{2}\right)\left(b_{r} e^{-i \Omega_{r} t}+b_{r}^{\dagger} e^{i \Omega_{r} t}\right) \\
& +\left\{\sum_{k, i=L, R} g_{k i} e^{i \omega_{k} t} c_{k i}^{\prime \dagger} d+f_{k i} e^{i \omega_{k} t} a_{k i}^{\prime} d+\text { H.c. }\right\}
\end{aligned}
$$

By introducing one bosonic coherent state and two fermionic coherent states as

$$
\begin{gathered}
|z\rangle=\prod_{r} \exp \left\{z_{r} b_{r}^{\dagger}\right\}|0\rangle, \\
\left|\xi_{i a}\right\rangle=\prod_{k}\left(1-\xi_{k i a} a_{k i}^{\prime \dagger}\right)|0\rangle(i=L, R), \\
\left|\xi_{i c}\right\rangle=\prod_{k}\left(1-\xi_{k i c} c_{k i}^{\dagger \dagger}\right)|0\rangle(i=L, R) .
\end{gathered}
$$

The stochastic state vector can be defined as

$$
\left|\psi_{t}\left(z^{*}, \xi_{L a}^{*}, \xi_{R a}^{*}, \xi_{L c}^{*}, \xi_{R c}^{*}\right)\right\rangle=\left\langle z^{*}, \xi_{L a}^{*}, \xi_{R a}^{*}, \xi_{L c}^{*}, \xi_{R c}^{*} \mid \psi_{t o t}(t)\right\rangle
$$

Following the general approach discussed in the last subsection, the NMQSD equation for the stochastic state vector is derived as

$$
\frac{\partial}{\partial t}\left|\psi_{t}\right\rangle=H_{e f f}\left|\psi_{t}\right\rangle,
$$

where

$$
\begin{aligned}
H_{e f f}= & {\left[-i H_{S}+d^{\dagger} \int_{0}^{t} d s K_{L a}(t, s) \frac{\delta}{\delta \xi_{L a, s}^{*}}+d \xi_{L a, t}^{*}\right.} \\
& +d^{\dagger} \int_{0}^{t} d s K_{R a}(t, s) \frac{\delta}{\delta \xi_{R a, s}^{*}}+d \xi_{R a, t}^{*} \\
& -d \int_{0}^{t} d s K_{L c}(t, s) \frac{\delta}{\delta \xi_{L c, s}^{*}}-d^{\dagger} \xi_{L c, t}^{*} \\
& -d \int_{0}^{t} d s K_{R c}(t, s) \frac{\delta}{\delta \xi_{R c, s}^{*}}-d^{\dagger} \xi_{R c, t}^{*} \\
& \left.-b^{\dagger} \int_{0}^{t} d s \alpha(t, s) \frac{\delta}{\delta z_{s}^{*}}+d z_{t}^{*}\right]
\end{aligned}
$$

In this equation, we introduced five noises as

$$
z_{t}^{*}=-i \sum_{r} z_{r}^{*} e^{-i \Omega_{r} t}
$$




$$
\begin{aligned}
& \xi_{i a, t}^{*}=-i \sum_{k} \xi_{k i a}^{*} e^{-i \omega_{k} t},(i=L, R), \\
& \xi_{i c, t}^{*}=-i \sum_{k} \xi_{k i c}^{*} e^{-i \omega_{k} t},(i=L, R),
\end{aligned}
$$

and the corresponding correlation functions are

$$
\begin{gathered}
\alpha(t, s)=\sum_{r} \lambda_{r}^{2} e^{-i \Omega_{r}(t-s)}, \\
K_{i a}(t, s)=\sum_{k} g_{k i}^{2} e^{-i \omega_{k}(t-s)} \quad(i=L, R), \\
K_{i c}(t, s)=\sum_{k} f_{k i}^{2} e^{i \omega_{k}(t-s)} \quad(i=L, R),
\end{gathered}
$$

Among the noises above, $z_{t}^{*}$ is a complex Gaussian noise, $\xi_{i a, t}^{*}$ and $\xi_{i c, t}^{*}$ are Grassmann Gaussian noises. They satisfy the following statistical relations

$$
\begin{gathered}
\left\langle z_{t}\right\rangle_{b}=\left\langle z_{t}^{*}\right\rangle_{b}=0, \\
\left\langle z_{t}^{*} z_{s}\right\rangle_{b}=\alpha(t, s), \\
\left\langle\xi_{i a, t}^{*}\right\rangle_{f}=\left\langle\xi_{i a, t}\right\rangle_{f}=\left\langle\xi_{i c, t}^{*}\right\rangle_{f}=\left\langle\xi_{i c, t}\right\rangle_{f}=0, \\
\left\langle\xi_{i a, t}^{*} \xi_{i a, s}\right\rangle_{f}=K_{i a}(t, s),\left\langle\xi_{i c, t}^{*} \xi_{i c, s}\right\rangle_{f}=K_{i c}(t, s) .
\end{gathered}
$$

Following the technique discussed in subsection 4.1, the time dependent operators $O$ and $Q$ are defined as

$$
\begin{gathered}
\frac{\delta}{\delta z_{s}^{*}}\left|\psi_{t}\right\rangle=O\left(t, s, z^{*}\right)\left|\psi_{t}\right\rangle, \\
\frac{\delta}{\delta \xi_{i a, s}^{*}}\left|\psi_{t}\right\rangle=Q_{i a}\left(t, s, \xi_{i a}^{*}\right)\left|\psi_{t}\right\rangle \quad(i=L, R), \\
\frac{\delta}{\delta \xi_{i c, s}^{*}}\left|\psi_{t}\right\rangle=Q_{i c}\left(t, s, \xi_{i c}^{*}\right)\left|\psi_{t}\right\rangle \quad(i=L, R),
\end{gathered}
$$

and the the zeroth order approximation gives the solution of these operators as

$$
\begin{gathered}
O \approx f_{1}(t, s) d^{\dagger} d, \\
Q_{i c} \approx f_{i c}(t, s) d(i=L, R), \\
Q_{i a} \approx f_{i a}(t, s) d^{\dagger}(i=L, R),
\end{gathered}
$$


while the coefficients satisfy

$$
\begin{gathered}
\frac{\partial}{\partial t} f_{1}(t, s)=0 \\
\frac{\partial}{\partial t} f_{L c}(t, s)=\left(i \varepsilon+F_{1}+F_{L a}+F_{R a}+F_{L c}+F_{R c}\right) f_{L c} \\
\frac{\partial}{\partial t} f_{R c}(t, s)=\left(i \varepsilon+F_{1}+F_{L a}+F_{R a}+F_{L c}+F_{R c}\right) f_{R c} \\
\frac{\partial}{\partial t} f_{L a}(t, s)=\left(-i \varepsilon-F_{1}-F_{L a}-F_{R a}-F_{L c}-F_{R c}\right) f_{L a} \\
\frac{\partial}{\partial t} f_{R a}(t, s)=\left(-i \varepsilon-F_{1}-F_{L a}-F_{R a}-F_{L c}-F_{R c}\right) f_{R a},
\end{gathered}
$$

where $F_{1}=\int_{0}^{t} \alpha(t, s) f_{1}(t, s) d s, F_{L c}=\int_{0}^{t} K_{L c}(t, s) f_{L c}(t, s) d s, F_{R c}=\int_{0}^{t} K_{R c}(t, s) f_{R c}(t, s) d s$, $F_{L a}=\int_{0}^{t} K_{L a}(t, s) f_{L a}(t, s) d s, F_{R a}=\int_{0}^{t} K_{R a}(t, s) f_{R a}(t, s) d s$. Finally, the master equation is derived as

$$
\begin{aligned}
\frac{\partial}{\partial t} \rho & =-i \varepsilon\left[d^{\dagger} d, \rho\right]+\left\{\left(F_{L c}+F_{R c}\right)\left[d \rho, d^{\dagger}\right]\right. \\
& \left.+\left(F_{L a}+F_{R a}\right)\left[d, d^{\dagger} \rho\right]+F_{1}\left[d^{\dagger} d \rho, d^{\dagger} d\right]+\text { H.c. }\right\}
\end{aligned}
$$

In the third example, the hybrid NMQSD approach is applied to a very important system called Anderson model embedded in a bosonic dephasing environment. First, we show how to map a finite temperature problem into a zero temperature problem to apply the hybrid NMQSD approach in finite temperature case. More important, we show the hybrid NMQSD approach provides us a powerful tool to investigate the dynamics of a quantum system in a non-Markovian regime. With the NMQSD approach, open quantum systems coupled to a hybrid bath such as the example discussed above can be solved systematically in non-Markovian regimes. Typically, in the Markov case, all the coefficients in the master equation, $F_{L c}, F_{R c}, F_{L a}, F_{R a}$, and $F_{1}$ are constants. However, in Eq. (106), those coefficients are time-dependent, which reflects the non-Markovian behavior even if the zeroth-order $O$ and $Q$ operators are employed. The higher-order non-Markovian approximations can be implemented in a similar way.

\subsection{Fermionic Bath vs. Bosonic Bath}

The master equation derived in Eq. (106) can be used to illustrate the difference between the fermionic bath and bosonic bath. For this purpose, two parameters in the original Hamiltonian are specified to describe the coupling strength to the fermionic bath and bosonic bath. As it is shown in Eq. (73), $t_{k i}$ determine how strong the interaction between the system and fermionic bath, and $\lambda_{r}$ determine the strength of the coupling to bosonic bath. In the 

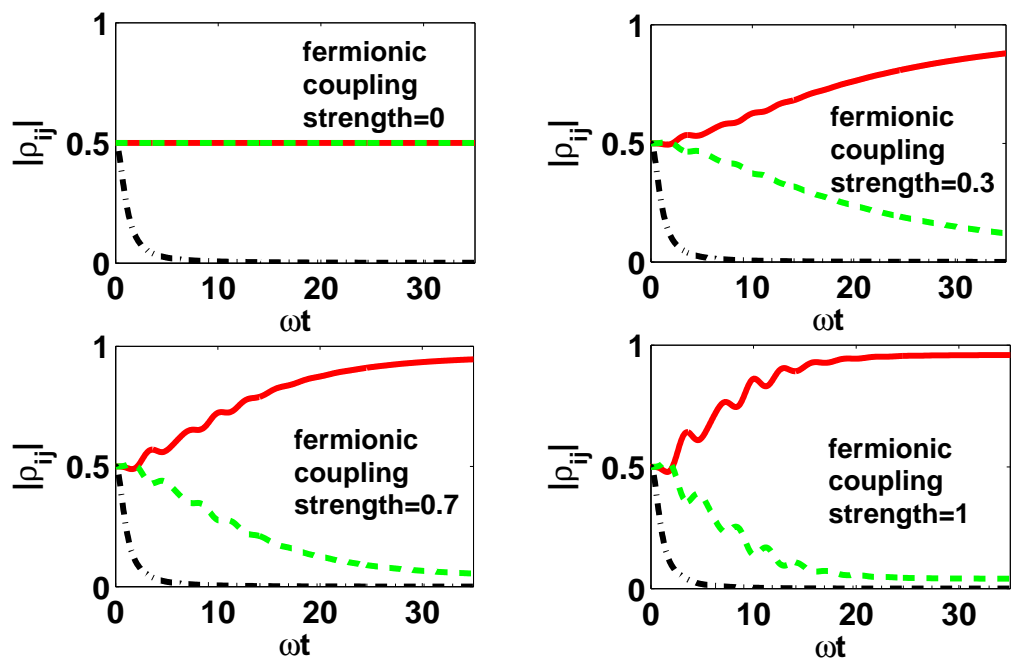

Figure 3: (Color online) Time evolution for different coupling strengths of fermionic bath. The coupling strength of bosonic bath is fixed as 1 . The red (solid), green (dashed), and black (dash-dotted) curves are the elements of density matrix $\left|\rho_{11}\right|,\left|\rho_{22}\right|,\left|\rho_{12}\right|$ respectively.

numerical simulation, we will introduce $c_{f}$ and $c_{b}$ to control the global coupling strengths for fermionic bath and bosonic bath respectively. Namely, we replace $t_{k i}$ by $\sqrt{c_{f}} t_{k i}$ and $\lambda_{r}$ by $\sqrt{c_{b}} \lambda_{r}$. Then, these two parameters reflect the global coupling strength. For example, if we take $c_{b}=0$, then the bosonic bath is switched off, and we can observe the evolution without presence of the bosonic bath. In the numerical simulations, we use four Ornstein-Uhlenbeck noises $K_{m n}(t, s)=\frac{\Gamma_{m n}}{2} \exp \left[\left(-\gamma_{m n}+i \phi_{m n}\right)|t-s|\right](m=L, R ; n=a, c)$ to model the correlation functions $K_{L a}, K_{L c}, K_{R a}, K_{R c}$. The parameters are chosen as $\Gamma_{L c}=0.017, \gamma_{L c}=0.3, \phi_{L c}=1.1, \Gamma_{R c}=0.034, \gamma_{R c}=0.5, \phi_{R c}=1.65$, $\Gamma_{L a}=0.012, \gamma_{L a}=0.4, \phi_{L a}=0.75, \Gamma_{R a}=0.044, \gamma_{R a}=0.45, \phi_{R a}=1.2$.

Fig. 3]and 4 clearly show the different influences of bosonic bath and fermionic bath on the system. Generally, For the model under consideration, the fermionic bath contributes to both of the energy dissipation and the decoherence, while the bosonic bath mainly contributes to the dephasing process. From Fig. 3 we can see that the dephasing process (off-diagonal elements) remains almost the same while changing the fermionic coupling strength. On the contrary, the dissipative process is significantly modified. From Fig. 4, we can see the dissipative process is not significantly affected by changing the bosonic coupling strength, as a compassion, the dephasing rate is affected. These results can be also predicted by analyzing the master equation or Hamiltonian. Since the coupling form of the bosonic bath is a dephasing type, therefore it will fundamentally affect the dephasing process. In a similar fashion, the coupling form 

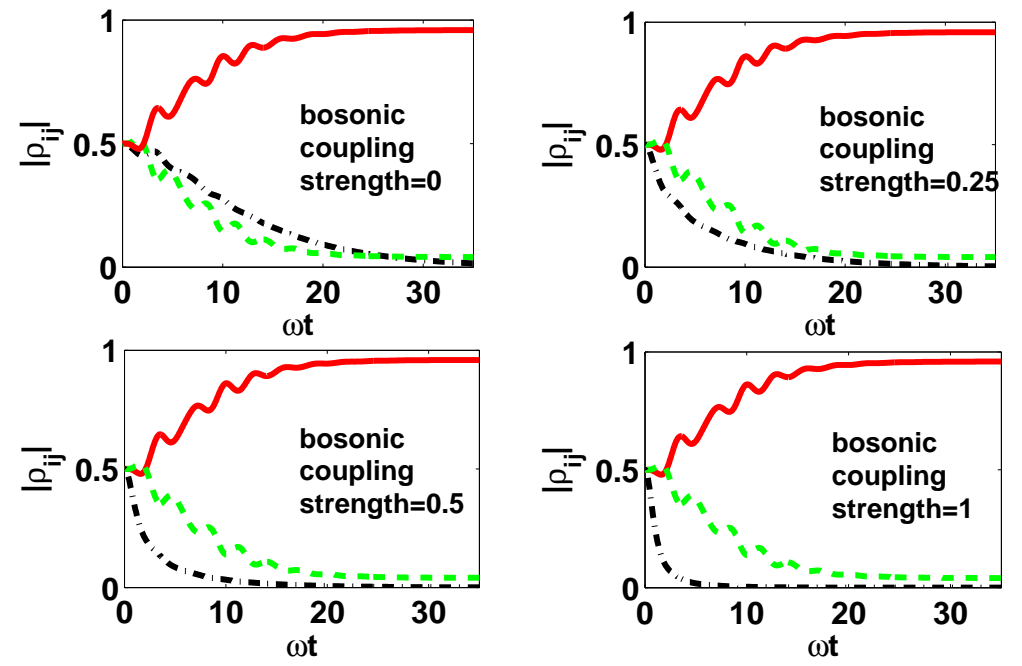

Figure 4: (Color online) Time evolution for different coupling strength of bosonic bath. The coupling strength of fermionic bath is fixed as 1 . The red (solid), green (dashed), and black (dash-dotted) curves are the elements of density matrix $\left|\rho_{11}\right|,\left|\rho_{22}\right|,\left|\rho_{12}\right|$ respectively.

of the fermionic bath is expected to affect the dissipative process.

\section{Conclusion}

In this paper, we have developed a stochastic Schrödingier equation for the open systems interacting with a hybrid environment containing both bosons and fermions. By combining the bosonic and fermionic NMQSD approaches, two types of noises are used simultaneously to derive the NMQSD equation for the hybrid bath case. As a simple application, we show that the corresponding non-Markovian master equation for the hybrid bath case can be recovered from the hybrid NMQSD equation. For more applications, two types of models are discussed including the commutative and anti-commutative cases. In these examples, we have demonstrated the consistency between NMQSD approach and the ordinary quantum mechanics when the system and its environment are relatively simple. Moreover, with these examples, we illustrate the relationship between bosonic bath and fermionic bath, the exact and approximate solutions of $O$ and $Q$ operators, and the different effects of bosonic bath and fermionic bath. The hybrid NMQSD approach established in this paper can be served as a convenient tool in the study of the dynamic evolution of the hybrid open systems. Particularly, it is helpful to investigate some early stage evolution caused by memory effect of the environments since our approach is systematically derived from the microscopic model which goes beyond the standard Born-Markov approximation. 


\section{Acknowledgements}

We acknowledge grant support from the NSF PHY-0925174, The NBRPC No. 2009CB929300,the NSFC Nos. 91121015 and the MOE No. B06011. J.Q.Y is supported by the NSAF (Grant Nos. U1330201 and U1530401) and the National Basic Research Program of China (Grant Nos. 2016YFA0301201 and 2014CB921401).

\section{References}

\section{References}

[1] X. Hu, R. de Sousa, S. Das Sarma, Foundations of Quantum Mechanics in the Light of New Technology (Edited by Y. A. Ono and K. Fujikawa, World Scientific, 2002).

[2] G. Ritschel, J. Roden, W. T. Strunz, A. Aspuru-Guzik, and A. Eisfeld, J. Phys. Chem. Lett. 2, 2912 (2011).

[3] N. Lambert and F. Nori Phys. Rev. B 78, 214302 (2008).

[4] C. W. Gardiner and P. Zoller, Quantum Noise (Springer-Verlag, Berlin, 2004).

[5] W. G. Unruh, Phys. Rev. A 51, 992 (1995).

[6] H. P. Breuer and F. Petruccione, Theory of Open Quantum Systems (Oxford University, New York, 2002).

[7] X. Zhao, J. Jing, B. Corn, and T. Yu, Phys. Rev. A 84, 032101 (2011).

[8] J. Jing, X. Zhao, J. Q. You, and T. Yu, Phys. Rev. A 85, 042106 (2012).

[9] W. H. Zurek, Rev. Mod. Phys. 75, 715 (2003).

[10] M. Schlosshauer, Rev. Mod. Phys. 76, 324 (2005).

[11] W. H. Zurek, S. Habib, and J. P. Paz, Phys. Rev. Lett. 70, 1187 (1993);

W. H. Zurek and J. P. Paz, Phys. Rev. Lett. 72, 2508 (1994).

[12] T. Yu and J. H. Eberly, Quantum Inform. Comp. 7, 459 (2007).

[13] T. Yu and J. H. Eberly, Phys. Rev. Lett. 93, 140404 (2004); Phys. Rev. Lett. 97, 140403 (2006).

[14] J. Jing, X. Zhao, J. Q. You, W. T. Strunz, and T. Yu, Phys. Rev. A 88, $052122(2013)$.

[15] X. Zhao, J. Jing, J. Q. You, and T. Yu, Quantum Inform. Comp. 14, 0741 (2014). 
[16] E. L. Hahn, Phys. Rev. 80, 580 (1950); W.-K. Rhim, A. Pines, and J. S. Waugh, Phys. Rev. Lett. 25, 218 (1970); L. Viola, E. Knill, and S. Lloyd, Phys. Rev. Lett. 82, 2417 (1999).

[17] H. M. Wiseman and G. J. Milburn, Phys. Rev. Lett. 70, 548 (1993); H. M. Wiseman, Phys. Rev. A 49, 2133 (1994).

[18] R. P. Feynman, and F. L. Vernon, Ann. Phys. 24, 118 (1963).

[19] J.-H. An and W.-M. Zhang, Phys. Rev. A 76, 042127 (2007); C. U. Lei, and W. M. Zhang, Ann. Phys. 327, 1408 (2012).

[20] B. L. Hu, J. P. Paz, and Y. Zhang, Phys. Rev. D 45, 2843 (1992); B. L. Hu, J. P. Paz, and Y. Zhang, Phys. Rev. D 47, 1576 (1993).

[21] B. L. Hu, J. P. Paz, and Y. Zhang, Phys. Rev. D 45, 2843 (1992); Phys. Rev. D 47, 1576 (1993).

[22] E. A. Calzetta, and B. L. Hu, Nonequilibrium Quantum Field Theory (Cambridge University Press, New York, 2008).

[23] A. O. Caldeira and A. J. Leggett, Physica A 121, 587 (1983).

[24] J. Dalibard, Y. Castin, and K. Mølmer, Phys. Rev. Lett. 68, 580 (1992).

[25] N. Gisin and I. C. Percival, J. Phys. A 25, 5677 (1992); ibid. 26, 2233 (1993).

[26] L. Diósi, N. Gisin, and W. T. Strunz, Phys. Rev. A 58, 1699 (1998); W. T. Strunz, L. Diósi, and N. Gisin, Phys. Rev. Lett. 82, 1801 (1999).

[27] T. Yu, L. Diósi, N. Gisin, and W. T. Strunz, Phys. Rev. A 60, 91 (1999).

[28] W. T. Strunz and T. Yu, Phys. Rev. A 69, 052115 (2004).

[29] Büttiker, Phys. Rev. B 46, 12485 (1992).

[30] Y. Meir, N. S. Wingreen, and P. A. Lee, Phys. Rev. Lett. 66, 3048 (1991); N. S. Wingreen, A.-P. Jauho, and Y. Meir, Phys. Rev. B 48, 8487 (1993).

[31] M. W. Y. Tu and W.-M. Zhang, Phys. Rev. B 78, 235311 (2008).

[32] W.-M. Zhang, P.-Y. Lo, H.-N. Xiong, M.W.-Y. Tu, and F. Nori, Phys. Rev. Lett. 109, 170402 (2012).

[33] X. Zhao, W. Shi, L.-A. Wu, and T. Yu, Phys. Rev. A 86, 032116 (2012).

[34] W. Shi, X. Zhao, and T. Yu, Phys. Rev. A 87, 052127 (2013).

[35] M. Chen and J. Q. You, Phys. Rev. A 87, 052108 (2013).

[36] T. Yu, Phys. Rev. A 69, 062107 (2004). 
[37] J. Jing and T. Yu, Phys. Rev. Lett. 105, 240403 (2010).

[38] D. Suess, A. Eisfeld, and W. T. Strunz, Phys. Rev. Lett. 113, 150403 (2014).

[39] Z.-Z. Li, C.-T. Yip, H.-Y. Deng, M. Chen, T. Yu, J. Q. You, and C.-H. Lam, Phys. Rev. A 90, 022122 (2014).

[40] H. Yang, H. Miao, and Y. Chen, Phys. Rev. A 85, 040101(R) (2012).

[41] J. Jing, L.-A. Wu, J. Q. You, and T. Yu, Phys. Rev. A 85, 032123 (2012).

[42] G. Ritschel et al., J. Phys. Chem. Lett. 2, 2912 (2011).

[43] E. Barouch, B. McCoy, and M. Dresden, Phys. Rev. A 2, 1075 (1970).

[44] W. -M. Zhang, D. H. Feng, and R. Gilmore, Rev. Mod. Phys. 62, 867 (1990).

[45] J. Xu, X. Zhao, J. Jing, W. T. Strunz, and T. Yu, J. Phys. A: Math. Theor. 47, 435301 (2014).

[46] C.-K. Chan, G.-D. Lin, S. F. Yelin, and M. D. Lukin, Phys. Rev. A 89, 042117 (2014).

[47] C.-H. Chung, K. Le Hur, M. Vojta, and P. Wölfle, Phys. Rev. Lett. 102, 216803 (2009); C.-H. Chung, Phys. Rev. B 83, 115308 (2011). 